\title{
NEW APPROACHES IN RESEARCH ASSESSMENT - FROM BIBLIOMETRICS TO GOALS-ORIENTED APPROACHES. THE CASE OF RESEARCHERS' ASSESSMENT FOR HIRING AND CAREER DEVELOPMENT IN ROMANIA
}

\author{
Ioana SPANACHE, PhD \\ Postdoctoral Researcher, National University of Political Studies and Public \\ Administration \\ Bucharest/ Romania \\ Alina IRIMIA, PhD \\ Open Science Knowledge Hub Coordinator, Executive Agency for Higher \\ Education, Research, Development and Innovation Funding \\ Bucharest/ Romania

\section{Adrian CURAJ, PhD} \\ Professor, UNESCO Chair on Science and Innovation Policies, National \\ University of Political Studies and Public Administration \\ Bucharest/ Romania
}

\begin{abstract}
In recent years, there has been increased attention given to how research assessment is conducted at different levels - research proposals, individual researchers, research organizations. In this context, the current paper explores existing literature regarding current research assessment approaches, and especially recommendations provided through four reference documents on the topic: the San Francisco Declaration on Research Assessment (2013), the Leiden Manifesto (Hicks et al. 2015), Science Europe's Position Statement and
\end{abstract}


Recommendations on Research Assessment Processes (2020), and the Hong Kong Principles for assessing researchers (Moher et al. 2020). The aim is to provide a basis for analysing the legislative and normative framework regulating how researchers are being evaluated in Romania for hiring and career development, and then apply it to identify recommendations regarding how the latter can be improved. Some of the recommendations identified refer to aspects such as: the need to explore evaluation arrangements which incorporate research activity related practices, and that measure performance against research units' research goals; increased focus on qualitative approaches, and on scientific content, as opposed to performance in publication metrics; developing a broader list with indicators, including Open Science and societal relevance related; introducing the principle of transparency; developing a monitoring and evaluation framework.

\section{Keywords}

Open Science; research assessment; research assessment practices in Romania; research careers; research performance.

\section{INTRODUCTION AND APPROACH}

In the last decade more and more principles and recommendations focused manifestos, essays and other types of documents have been developed saying how research assessment should change, both at the level of metrics and indicators used, as well as regarding the overall approach. However, we believe that such a change requires going beyond evaluation practices and used metrics, as changes are also needed in how we understand success in research and what good research performance means. Moreover, the way in which we define these concepts influences the choice of assessment approach and of indicators to be used. We evaluate research for different purposes and in different instances. We can evaluate research proposals or projects for funding, we evaluate researchers for career advancement, and Research Performing Organizations (RPOs) and their research activities. When it comes to the reasons for conducting such 
evaluations, research assessments can be done, for example, for accountability reasons, to allocate limited resources, or for improvement, to make research perform better in relation to the purposes of the programs that fund it, etc.

In this context, the paper first looks at existing literature and discussions regarding aspects such as how research performance and success are understood, approaches used and recommended for conducting research assessments, and their associated indicators and metrics, to get a clearer picture about recent developments and existing debates regarding the topic.

And afterwards, we took as reference four intensely debated documents with principles and recommendations - the San Francisco Declaration on Research Assessment (2013), the Leiden Manifesto for Research Metrics (Hicks et al. 2015), Science Europe's Recommendations on Research Assessment Processes (2020), and the Hong Kong Principles for assessing researchers: Fostering research integrity (Moher et al. 2020); to serve as guidance in analysing research and researchers' assessment practices used at national level, in Romania. The recommendations identified through the four documents were then used as a lens to look at the specific case of the evaluation methodology for evaluating researchers for hiring and career advancement as senior researchers (Scientific Researcher I and II). The aim was to draw conclusions regarding how existing methodology compares to the new approaches, what improvements and adjustments can be made, and what could the next steps be.

\section{RESEARCH PERFORMANCE AND SUCCESS DEFINITIONS AND DEBATE}

Before looking at how research, RPOs or researchers are being evaluated, we need to first look at how concepts such as success, performance, excellence, and quality in science are defined, as their understanding determine the choice of indicators and how they are built. On the other hand, such terms are difficult to define (Bonn and Pinxten 2021, 1), and numerous research funding organizations do not have, for example, a formal definition of what research 
quality means (Science Europe 2020, 10). Quality in research can be extremely context-dependent, a fact which can lead in certain cases to avoiding having a formal definition in this regard.

In this case, how can we determine if a research organization, be it a research institute or university, performs well? What about a researcher? Can we say that a high performing organization is the sum of high performing researchers?

According to a study conducted by Bonn and Pixten which involved consulting different actors from the research system, success in research was identified as being "a multi-factorial, context-dependent, and mutable construct" (2020, 1). Thus, the results of the study illustrated success in research as being determined by an intersection between characteristics of researchers (such as levels of skills and expertise, personality traits, status and network), outputs (publications, past successes, impact metrics, social value and applicability, teaching etc.), processes (the way researchers work, which can contribute to success irrespective of the final outputs e.g. collaborations/ multi-disciplinarity, the use of appropriate methodology, adherence to ethical standards, reproducibility, having a good research idea etc.), and other factors, such as luck (Bonn and Pixten 2021, 8), which can also be understood as a favourable context.

success in research

$=$ characteristics of researchers $\bigcap$ outputs $\bigcap$ processes $\bigcap$ favorable context

Bazeley, on the other hand, defined research performance, in a context in which she underlined the lack of an academic debate about what the concept means (2010, 890), as being composed of two elements - research (the activity), plus performance (the verb to perform) - 'what is learned through the research must be made visible and passed on (performed) to others' $(2010,897)$. In other words, the latter refers to making research results (and not only its results, as we will later argue) visible and available to others to use and build upon it. This definition can make us think that a good performance of research is equivalent to making a research activity and its results as visible as possible, so that it can be used by others. 


\section{research performance}

\section{$=$ research (activity) \\ + performance (making now knowledge visible \& passing it on)}

To what concerns the second component, performance, the two dimensions suggested by Bazeley are described as follows: dissemination being the formal communication of research outcomes - research 'is made visible so that others can benefit from it' $(2010,898)$; collegial engagement - the sharing of knowledge and expertise - 'sharing one's expertise (substantive or methodological) with others collegially, or in a leadership or supervisory capacity' (899) as a means of passing on knowledge. Moreover, when it comes to dissemination Bazeley $(2010,898)$ mentions publishing, perceived as a primary means, and conferences and seminars. However, in recent years, dissemination of research has started to embrace other forms also, facilitated by new technologies and platforms.

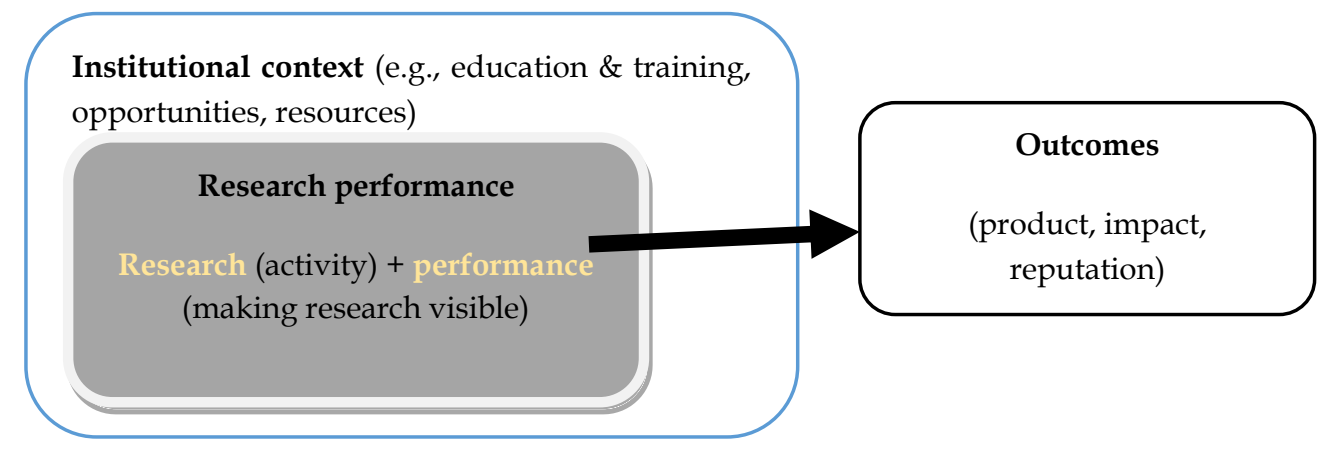

Figure 1. Authors' representation of Bazeley's definition of the research performance concept (2010)

Even though when referring to quality in research, more research organizations give higher importance to aspects such as novelty/ originality, methodological and theoretical rigor, and academic significance or impact (Technopolis Group 2019, 21), there are also instances in which it is encouraged that research performance and success should be strongly related and measured against the research goals of institutions, research groups or individual researchers, or in 
which it is recommended that research excellence should be also perceived as being locally relevant (Leiden manifesto 2015, 430).

Moreover, the essay "The Hong Kong principles for assessing researchers" advocates in this regard for a stronger focus on research integrity, as opposed to aspects such as novelty or academic impact (Moher et al. 2020, 2). And research integrity is seen by the authors as a precondition necessary for knowledge to benefit research and society. This differs to a certain degree from the approach proposed by Bazeley (2010), who linked research performance more to making research outcomes visible.

The way success and performance in research are perceived depend on aspects related to research culture and are in strong relation with the criteria and indicators used in research assessments. The former determines the latter, and the latter can give us information about how the former is being understood, in cases in which formal definitions are missing. Either way, in the existing literature we notice a diverse understanding of what research performance and success mean - varying from being linked to research outputs, levels of originality and novelty, academic impact, to newer approaches that encourage a focus on research integrity and responsible practices ${ }^{1}$, on societal impact, or measuring it against institutional or researcher's goals.

\section{RESEARCH ASSESSMENT}

Research assessment can be used in different instances, such as for the evaluation of research proposals and projects for funding, evaluation of researchers for career advancement or evaluation of research organizations and of their research activities. In this section we look at different research assessment approaches (e.g., predominantly quantitative, or qualitative), depending on what they are focused on, in what concerns used criteria, metrics,

1 Such as in the case of the Hong Kong principles (Moher et al. 2020) 
and indicators, together with associated advantages and disadvantages, as well as their known or potential effects.

The focus of research assessment depends on what is considered as being important and relevant by the research community, decision-makers, and research organizations. The former matters because it not only depends on what is considered initially as being important, but it also leads to further influences regarding what is rewarded and incentivized, thus leading to changes in researchers' and research organizations' behaviour. Moreover, research assessment purposes can refer to accountability, in a context of limited resources and of making sure that those available are spent with efficiency and effectiveness, or to learning and improvement, for quality assurance. If we follow the logic from the definition provided by Bazeley $(2010,897)$, we also reach a certain understanding of how research assessment should be performed. In this context, we understand that evaluation processes need to focus on how visible a research activity and its results are, as well as on how much they were used by others.

\subsection{Quantitative approaches}

A JRC study conducted in 2016 regarding Research Performance Based Funding Systems (RPBF), identified that numerous countries use for allocating funding under such systems formulae based partially on a quantitative assessment of research outputs (Jonkers and Zacharewicz 2016, 6). The rest use either peer review to evaluate research outputs, or a mix between the two approaches, in which quantitative assessment are used to inform the peer review process.

From the countries implementing RPBF systems, Belgium, Czech Republic, Denmark, Estonia, Finland, Croatia, Poland, Sweden and Slovakia were using at the time quantitative formulas with bibliometric assessment as a dominant assessment approach, while France, Italy, Lithuania, Portugal and the United Kingdom were using peer review as a dominant approach instead. (Jonkers and Zacharewicz 2016, 21) 
Bibliometrics involve the statistical analysis of the academic impact generated by research outputs such as articles, books, and other types of publications. These types of metrics can be measured at the level of articles (e.g. citation counts), journals (i.e. journal impact factor, the h-index, acceptance rate, and other), and author (i.e. h-index - this time tracking impact of the research output at the level of a single researcher, g-index, i10-index - created by Google Scholar).

Even though bibliometrics were and still are in certain instances preferred because of their perceived objectivity, reliability, and cost-effectiveness (Campbell et al. 2010), in recent years, more and more authors (Bazeley 2010; Hicks et al. 2015; Moher et al. 2020; Science Europe 2020 and others) have argued against the use of some of the associated metrics, e.g., journal impact factor, other journal-based metrics, and even against all publication related metrics. In this context, Bazeley (2010) talked about expediency, and the fact that the large use of bibliometrics is a result of the fact that a primary driver in determining what to measure refers to how easy and practicable is to collect the needed data (890). Similarly, Moher et al. consider that, frequently, research assessments end up concentrating on a "narrow range of easy-to-measure metrics, including publications, citations, and funding income" $(2020,8)$.

However, some of the disadvantages with which this kind of metrics and quantitative approaches are associated refer to aspects such as a false perceived precision - in the case of the impact factor and the number of decimals provided for them (Hicks et al. 2015, 431), not taking into consideration the variations existing between research fields (e.g., no. of citations varies profoundly between Biology and Humanities), gaming practices, such as "salami slicing", artificially splitting research into different articles to increase their number (Elsevier 2019), and maybe most importantly, the fact that they do not always reflect quality in research, but rather the attention the latter receives in some instances. 


\subsubsection{Altmetrics}

Additionally, because of new technologies appearing, new metrics have been suggested for measuring the perceived impact of scientific publications. This is the case of altmetrics, the short name for alternative metrics, which are based mainly on social media platforms (e.g., Twitter, ResearchGate, Mendeley, etc.) which measure signals such as the no. of shares, likes, downloads, followers, posts, mentions and comments (European Commission 2017, 9-10). They are part of the bibliometrics family, and they are used in complementing traditional metrics.

Advantages of altmetrics, as described by Priem et al. (2010) refer to their extended coverage, as they can be used not only in the case of journal publications, but also for other types of research outputs and materials such as databases, code, designs, blog posts and other; as well as to their diversity, by offering multiple ways of tracking the impact of a single research object downloads, likes, comments etc. However, altmetrics are also associated with certain disadvantages or challenges that make us question them, starting with their lack of robustness and the ease with which they can be gamed. Other disadvantages refer to the lack of free access to their underlying data, as they belong to the commercial actors managing the respective social platforms, as well as to the gaps in knowledge related to how these altmetrics function, as they are dependent on aspects such as people's behaviour in terms of liking and sharing, their underlying motives for sharing, and the types of users registered on social media platforms (European Commission 2017, 12).

As a response to some of these challenges, certain platforms have started collecting their own data for measuring altmetrics. This is also the case for the catch-all repository Zenodo, which tracks its own data for metrics such as no. of (unique) views and no. of (unique) downloads and makes it available on each research object's page. 


\subsection{Qualitative approaches}

As opposed to using bibliometrics and quantitative approaches in research assessment, some countries and funding organizations use predominantly qualitative approaches based on peer review: e.g., UK, France, Italy, Lithuania, Portugal (Jonkers and Zacharewicz 2016, 21). And the most frequent example provided in this regard refers to the case of the Research Excellence Framework (REF), the system used in the UK for assessing research quality in higher education organizations (REF official webpage). REF is implemented through a process of expert review conducted by expert panels set up for each unit of assessment. And to get a better idea about the type of practices that characterize qualitative approaches, let us look at the type of criteria and instruments used. In the case of REF, the evaluation criteria refer to three main elements - the quality of outputs, their impact beyond academia and the environment supporting research. Moreover, UKRI, one of the funding organizations which undertake the REF, has recently announced that it will introduce a new and single format for researchers' CV across all its programs, a narrative one, that aims to consider a wider range of research outputs, skills and research activities (UKRI webpage).

In the study conducted on 39 research organizations by the Technopolis Group for Science Europe, it was identified that most participating organizations have indicated that they are currently using qualitative approaches for assessing research outputs when evaluating applicants for funding, researchers for career advancement and, in some cases, research institutes $(2019,18)$. This practice is also gaining more and more attention, as it is also encouraged by different reference documents, such as the San Francisco Declaration on Research Assessment, and as an increasing number of organizations are reducing their use of journal-based metrics.

Some advantages of using qualitative approaches refer to the introduction of the human factor, for example, research outputs being judged on a case-by-case basis by panels of reviewers, as well as to the fact that they allow considering differences existing between research fields, in which case bibliometrics might 
be subject to limitations. In contrast, some identified disadvantages refer to higher costs, risk of subjectivity and the exhaustion of the pool of reviewers, as indicated by the Technopolis Group $(2019,16)$. To tackle potential disadvantages and limitations, certain countries and organizations use, as different reference documents recommend (Leiden Manifesto 2015, 430), a mixed approach, in which quantitative evaluation approaches support qualitative, expert assessment.

\subsection{Evaluation criteria}

Evaluation criteria was identified in a study conducted in 2019 mostly on research funding organizations (Technopolis Group, 4) as being dependent on the objectives of funding, as they are different in the cases in which we talk about discipline-based evaluation versus the cases in which they are used in problem-oriented evaluations.

When it comes to research assessment criteria, in the last approximately 70 years we have been experiencing three different phases, which were observed as overlapping in the case of certain research systems in the study conducted by the Technopolis Group for Science Europe $(2019,6)$. The first phase, which started in 1945, and in which the scientific community was considered as being the most appropriate actor for taking decisions regarding what topics should be researched or not, the assessment criteria focused mainly on scientific quality. In 1960, when the OECD has argued for the need to direct research towards socially determined goals, assessment criteria has started focusing along scientific quality, also on societal relevance, as part of the second phase. In the third phase, starting from 2000, the need for tackling societal challenges through research was reinforced, assessment criteria becoming more strongly linked to higher-level strategies. In this context, the study identified that all Science Europe member organizations deal with assessment criteria corresponding to the first phase, while a numerous part of them deal also with the second phase type of criteria, and only a few consider criteria related to the third phase - by responding to societal challenges. 


\subsection{Indicators and metrics}

When it comes to indicators and metrics, two main topics were identified in the principles and recommendations offered by the four documents (DORA, Leiden Manifesto, Science Europe recommendations, Hong Kong principles). The first one refers to how journal-based metrics should be approached. And recommendations in this regard vary from the complete removal of journalbased metrics, such as the impact factor (Hicks et al. 2015), to acknowledging their limitations and valuing more the scientific content of research papers over their associated metrics or the identity of the journals hosting them (DORA 2013) to giving less importance to all research outputs related indicators in general (Moher et al. 2020).

In this regard, from the 39 RFOs and RPOs consulted by the Technopolis Group in 2019,16 (41\%) declared that they have either made the change or plan to do so, in the case of reducing the use of journal-based metrics, while 13 (33\%) declared the same in the case of a complete elimination of such metrics (Technopolis Group 2019, 19). These responses were recorded in a context in which, a little over half of respondents are DORA signatories.

Further, the second topic refers to having a suite of indicators and metrics reflecting both research outputs and best practices in research processes and activity, such as data sharing. One example in this sense is provided by the Strategy Evaluation Protocol 2021-2027, which is the guiding document for evaluating all research units from the Netherlands. In its case, an indicative and flexible list of indicators and metrics is provided, from which research units can choose the ones that best suit their aims and strategies.

By having a suite of indicators and metrics we can allow for a better acknowledgement of the variations existing across fields and types of research (Hicks et al. 2015, 430). This involves the use of indicators that reflect a wider range of research outputs and activities, outside of publication metrics. And here, the principles and recommendations refer to four important aspects:

1. indicators and metrics need to be diverse enough to reflect the variations existing between research fields and citation practices (Hicks et al. 2015, 430); 
2. consider other types of research outputs, more qualitative ones, plus going beyond publication metrics (DORA, 2013);

3. develop and incorporate indicators and metrics related to research activity and process related practices, not only research outputs - e.g., indicators related to accurate and transparent reporting of research, irrespective of research results (Moher et al. 2020);

4. develop indicators that reflect different types and stages of research (Moher et al. 2020, 7).

Here, some interesting examples refer to the Researchfish platform used by UKRI (United Kingdom Research and Innovation), the largest research funding body in the UK, and by other research funding and performing organisations for tracking research impact in a more comprehensive and diverse way. Moreover, UKRI is also currently working on developing format of researcher resume to be used in research assessment, a narrative format that goes beyond traditional academic CVs formats and tries to capture a wider range of research skills and contributions (UKRI 2021). UKRI is also one of the most prominent member organisations of DORA.

And if we put these observations in the context of the previous dimension, regarding recommended assessment approaches, the indicators and metrics used within a system, irrespective of how diverse and comprehensive, they are meant only to support and inform more qualitative analyses.

\subsection{Research assessment and Open Science principles and practices}

Open Science represents a new paradigm regarding how research is being conducted, shared, and assessed, which has started producing more and more changes on research culture. After conducting a systematic literature review to formulate an integrated definition of the Open Science concept and phenomenon, Vicente-Saez and Martinez-Fuentes $(2018$, 433) reached the following conclusion: "Open Science is transparent and accessible knowledge that is shared and developed through collaborative networks". 
By looking at other four different definitions given to Open Science (UNESCO 2020; European Commission 2016; OECD 2015; Foster n.d.), we notice that the concept involves the sharing and using of research outputs and materials even from an earlier stage, by making them publicly available, to enhance collaboration and accelerate research. Among the elements that constitute Open Science, as an umbrella term, are open access to publications, open access to data, and even to research notes, citizen science, open source software, and other.

Moreover, according to Wouters et al. $(2019,6)$ Open Science is not only about making knowledge available without any restrictions. Rather, it requires an indepth analysis of the diversity of scientific practices, whether they are oriented towards scientific progress as an objective in itself, or towards solving societal challenges, or a combination of the two.

To understand better what can be achieved through Open Science practices, let us look at one of the most well-known and relevant examples in this regard the Human Genome project. The project started in 1990 at the initiative of the United States, but later became the largest collaborative biological project in the world, by involving five other states - UK, France, Germany, Japan, and China. During a meeting that took place in Bermuda in 1996, representatives from participating organizations adopted a set of principles regarding the free availability of all human genomic sequence information, meant at maximizing its benefit to society, as well as regarding the fact that all produced data must be released as soon as possible and daily through public databases (Human Genome Project Information Archive 1990-2003). Later studies on the economic return of the project have shown that, for each 1 US Dollar spent on the project, other 141 US Dollars were generated (Tripp and Grueber 2011, 2).

In this context, a working group within the European Commission' $\mathrm{s}$ Directorate-General for Research and Innovation has drawn up a report with proposals and recommendations on indicator frameworks to promote open knowledge practices in science and academia. As it also happens in other cases, the indicators that will be used in research assessment can exercise a great influence on the form that Open Science will take, as they can influence both the decisions regarding science policy and the ones regarding the agreed way of 
generating new knowledge (Wouters et al. 2019, 3). Thus, the authors propose four sets of indicator toolboxes, together with some information needed for their application. The four sets are organized into categories in accordance with four potential pursued purposes as part of an evaluation process: monitoring - the extent to which the scientific system makes the transition to a more open and inclusive mode of operation; learning - relevant in formative assessments and closely related to the research context; resource allocation and career evaluation of researchers - (summative evaluation) comparative indicators for assessing the performance of institutes and research groups in order to allocate resources (Wouters et al. 2019, 6-8).

Some examples of such indicators, that aim at fostering open knowledge practices, are: attitudes of researchers towards data sharing, no. of publications that can be tracked through different almetric sources (e.g. DOI, Scopus id etc.), no. of citizen science projects, percentage of researchers in citizen science programs, \% of funded projects incorporating costs for data compilation, publication and maintenance, $\%$ of researchers that share data, $\%$ of machinereadable data or metadata, $\%$ of open access publications, no. of institutes with OA repositories, no. of tweets to publications from highly followed tweeters etc. (Wouters et al. 2019, 22-28). As can be seen from the examples listed above, the proposed indicators can refer to different dimensions such as citizen science, data sharing adoption, machine-readable data and metadata, adoption of Open Access practices, science communication and other.

\section{NEW APPROACHES IN RESEARCH ASSESSMENT}

As previously mentioned, the article takes an in-depth look at the key recommendations provided through four frequently used and cited documents regarding research assessment: the San Francisco Declaration on Research Assessment (2013), the Leiden Manifesto for Research Metrics (Hicks et al. 2015), Science Europe's Recommendations on Research Assessment Processes (2020), and the Hong Kong Principles for assessing researchers: Fostering research integrity (Moher et al. 2020). For this, we have clustered the identified 
recommendations around five dimensions/ topics of interest: meaning of research performance, assessment approach, metrics and indicators, transparency, revision of assessment processes. And for each of them we have provided guiding questions to serve in the analysis of research assessment practices, regardless of the country in which they are implemented.

\subsection{Research performance}

In this regard, the Leiden Manifesto offers guidance by recommending that performance of research organizations, groups or individual researchers be measured against their own research goals and missions. One example in this sense is offered in the Netherlands, where the Strategy Evaluation Protocol 20212027 involves that each research unit is evaluated in light of its own aims and strategy. Moreover, the document also advocates for taking into consideration the local relevance of research, as its perceived level of excellence is strongly context dependent. Here, we can think of cases from the Humanities, from Sociology or Anthropology, where given the national or regional focus of certain topics, the resulting research outputs might not achieve as much academic impact as research from other fields, e.g., Biology, that has a more global focus. And, as it was previously described in the Research performance and success section of this paper, the Hong Kong principles (Moher et al. 2020) try, when it comes to what good research performance means, to put a stronger focus on research practices, their integrity, rigor, and transparency as opposed to research outputs.

\subsection{Assessment approach}

Two out of the four reference documents explicitly advocate for using a qualitative approach. The Leiden Manifesto recommends the implementation of a predominantly qualitative assessment approach in which quantitative evaluation supports qualitative, expert assessments, and in which portfolios of individual researchers are judged from a qualitative point of view (Hicks et al. 
2015, 430). Meanwhile, Science Europe advocates for qualitative assessment processes that enable evaluations to focus on content and that consider a wide range of research outputs and activities $(2020,7)$.

\subsection{Metrics and indicators}

A broad description on how metrics and indicators are approached in the four documents is provided in the dedicated section from above.

To sum up, the documents include recommendations regarding two main topics. The first one refers to how journal-based metrics should be approached, and the recommendations in this direction range from the complete removal of such metrics (Leiden Manifesto 2015), to acknowledging their limitations and downsides (DORA 2013), and giving less importance to all publication metrics entirely, by prioritizing instead the scientific content of research papers (Hong Kong principles, Moher et al., 2020). And the second one refers to having a suite of indicators and metrics reflecting both research outputs and best practices in research processes and activity. This allows for a better acknowledgement of the variations existing across fields and types of research (Leiden Manifesto 2015). And it involves the use of indicators that reflect a wider range of research outputs and activities, outside of publication metrics. Under this topic, four specific recommendations were identified and are listed in the previously mentioned section.

\subsection{Transparency}

In addition to the topics identified before, the analysis revealed recommendations addressing topics such as transparency and the need to periodically revise assessment processes. In what concerns transparency, the Leiden Manifesto recommends for data collection and analysis processes (part of research assessment processes) to be open, transparent, and simple. Moreover, the document encourages that evaluated candidates have access to the results, as 
well as to the underlying data and analyses (Hicks et al. 2015, 430). Before that, DORA (2013) recommended that the evaluation criteria used in making decisions about hiring, tenure, and promotion of researchers and academic staff to be presented as explicitly as possible. And Science Europe $(2020,11)$ encourages that research assessment processes are made clear and transparent during the entire process of implementation.

\subsection{Revision of assessment processes}

Research assessment practices and indicators can lead to effects on the behaviour of individual researchers and of research units, as well as on the entire research system. Thus, it is necessary that these kinds of effects are recognized and periodically investigated. For this, indicators must be tested and their (potential) effects analysed regularly. (Hicks et al. 2015, 431)

Science Europe recommendations go further, and beside encouraging the evaluation of research assessment processes' robustness, it makes references to the sharing of best practices identified during the monitoring and evaluation of those processes $(2020,7)$. Moreover, the organization also advocates for giving special attention to discrimination, bias and unfair treatment in this regard, and that research organizations need to publicly show how they address this kind of potential situations in research assessment processes.

Other recommendations relevant in the context of this paper refer to: improving efficiency for all parties involved in research assessment, as well as improving cost-effectiveness; broadening the pool of reviewers by using broader selection criteria; research organizations considering novel approaches to research assessment, and the sharing of best practices identified in this regard. (Science Europe 2020, 7) 


\section{RESEARCH ASSESSMENT IN ROMANIA - THE CASE OF RESEARCHERS' EVALUATION FOR HIRING AND CAREER DEVELOPMENT}

In Romania, the evaluation of research and development staff from research institutes is regulated through several legislative and normative acts, such as Law no. 319/ 2003, which contains the main guiding principles regarding the status of research and development staff, Research Minister' Orders no. 5100/2005 and no. 5101/2005, which provide further information regarding the preliminary conditions and evaluation criteria that a candidate must comply with to become a senior researcher, as well as the later Minister's Orders from $2011^{1}, 2012^{2}$, and $2016^{3}$ regarding the approval of the minimum necessary and mandatory standards for conferring didactic titles in higher education, $R \& D$ positions and the habilitation certificate, for different scientific fields. According to the respective law, the evaluation of professional performances of candidates for hiring or career development is made by considering aspects such as: previous studies, level of competence, professional results obtained, scientific titles and professional degrees obtained, experience accumulated on the job, and the specific skills necessary to fulfill the position (Article 16 (1)).

Otherwise, the law in question includes explicit information only regarding the minimum necessary requirements in terms of number of years of professional experience that a candidate must have for hiring or career development, and information about the procedures needed for organizing such competitions.

In addition to these, each $R \& D$ unit can establish its own evaluation criteria for employment and award of a professional degree, as they are also the ones organizing the competitions. Subsequently, the results of competitions can either be confirmed or refuted by the National Council for Attestation of University

1 Orders no. 4478, 4691 and 4692 /2011

2 Order no. 6560/ 2012

${ }^{3}$ Order no. 6129/ 2016 
Degrees, Diplomas and Certificates (CNATDCU) based on an analysis, in the case of senior researchers.

Two years later, Orders no. 5100 and 5101 have provided further information regarding two preconditions with which candidates must comply, as well as about evaluation criteria. The two preconditions refer to 1 . national visibility and impact; and 2. international visibility and impact, the latter being measured through ISI-listed publications and/ or publications indexed in international databases relevant for the field, as well as contracts and grants obtained in international competitions. When it comes to evaluation criteria, figure no.1 below resumes them:

\section{Management of research projects/ activities}

- capacity to coordinate research projects and teams as principal investigator

- self-assessment + peer review by the department/ institution's management

\section{Research activity}

- scope and scale of RDI projects for which the candidate obtained funding (min. 3 research grants/ contracts, national and international)

\section{Scientific contribution}

- with a focus on scientometrics, e.g., no. of ISI articles, or articles indexed in international databases, books, chapters in books, studies; + no. of patents

- *ISI articles are favoured

\section{Professional prestige}

- 3 most significant scientific contributions brought to the research field and the presentation of a research contract/ grant.

- E.g., citations of ISI articles - perceived as evidence of recognition of achievements, coordination of professional scientific structures, affiliations to recognized international and national professional

Figure 2. Criteria for evaluating senior researchers 
And in 2011, a more detailed list with specific criteria and indicators was first provided through Minister's Orders no. 4478, 4691 and 4692. As opposed to the previous normative and legislative documents, in the case of the latter, we noticed an increased focus on the international dimension of research, as new indicators were introduced, and all of them are more explicitly described: e.g., having published at least one book by a prestigious international publishing house abroad in an international language, serving as a keynote speaker at international conferences organized abroad or in the country, associate or visiting professor at a university from abroad, editor for an academic journal from abroad or within the country, coordinator of a collection/ series of volumes published by a prestigious international publishing house etc. Otherwise, the criteria and indicators from the normative documents from both 2005 and 2011 are similar, with the distinction that in 2011, they are more specific and that they also include clear references to the impact factor, as candidates are scored differently depending on the impact factor of their published articles.

Later, additional changes were brought as a result of an endeavor of aligning national standards to relevant international ones, as well as for combating scientific fraud encountered when dealing with citations and the impact factor. In all normative documents from 2011, 2012, and 2016, each thematic committee established its own list of minimum and mandatory standards, along with certain guiding principles. Another reason for introducing a new Order of the Minister in 2016 refers to the need of aligning the standards between scientific disciplines, for each thematic committee.

Regarding guiding principles, certain committees have explicitly mentioned that the evaluation must reflect the level of involvement of the candidate in the activity of scientific research, technological development and innovation, in the didactic-professional activity, as well as the impact and recognition of his/ her involvement in these activities, at national and international level (Mathematics and Informatics Committees, Annex no.1, Order no 6129/2016). Thus, the Order recommends for evaluators to first consider the quality of the research conducted by candidates, as the list with minimum standards represents only a 
precondition for entering the competition, and one's compliance with the standards included are not sufficient to obtain the title.

However, for a more complete picture regarding the dynamic and reasons behind changes produced, further research needs to be conducted, which could include interviewing policy-makers and R\&D staff.

To what concerns the competition procedure, candidates must include in their file a presentation of studies, positions, and professional-scientific achievements, organized according to the evaluation criteria, and accompanied by the following two: a self-assessment of the candidate, containing his/ her own assessment on the fulfillment of the evaluation criteria, with references to each evaluation criterion, and a portfolio with publications; as well as the evaluation sheet from the management of the department or the institute regarding the quality of the research management activities, as described in the first criterion. Moreover, according to Law no. 319 from 2003, the assessment process consists of verifying if the necessary conditions are fulfilled and of giving points, based on a grid adapted to the specifics of the job activity and the candidates' performances. (Article 16 (2) c).

According to Article 16 of the respective Law (319/2003, (2) c), in the case of evaluating candidates for hiring or career advancement as senior researchers, the competition committee is composed of the scientific director or scientific secretary of the institution/ research unit and four members, university professors and senior researchers, of which at least two need to be from outside the institution or unit. The competition committee evaluates candidates' files and produces reports for all of them. Then, the president of the committee presents the reports to the scientific council of the research unit and nominates the candidate with the best results. Afterwards, the scientific council approves the competition's results by roll call vote. The competition files, together with the committee's reports are sent to CNATDCU, who needs to validate the competition's results. In this process, the final say on the matter belongs to the state authority for research and development who has the role of confirming the results. Then, the candidate is appointed as a senior researcher through an official decision of the head of the research institution/ unit who organized the competition. 


\section{KEY QUESTIONS AND KEY RECOMMENDATIONS}

Further, for drawing conclusions regarding how researchers are assessed in Romania, and providing recommendations for improvement, we have analysed the previously described procedures and methodology as opposed to the recommendations identified in the four reference documents. For this, we have used for each approached topic a set of guiding questions. For example, in the case of how research performance is understood, the key guiding questions formulated are: How is research performance understood in the analysed research assessment practices? What type of criteria are being used in research assessment processes? What is the research assessment focused on?

Compared with the normative documents from 2005, the ones regarding the minimum standards from 2011, 2012 and 2016 do not include a general set of evaluation criteria. But if we look at the criteria and principles mentioned by certain CNATDCU thematic commissions or at used indicators, we notice that performance in research is perceived as a combination of research outputs, academic impact (citations, visibility of research outputs), research management and funding obtained for research projects, as well as teaching related outputs in the case of teaching positions (courses, initiation of new study programs). However, from these, special focus is given to research outputs, especially articles published in ISI indexed journals, as well as to academic impact, especially citations.

The evaluation methodology described within the analysed legislative and normative documents does not include any references regarding a potential special focus on research activity and reporting related processes, such as integrity, rigor, or transparency, as recommended by the Hong Kong principles (Moher et al. 2020), but in some cases, they include mentions regarding research ethics as an evaluation criterion or even as a precondition for considering a candidate for the position.

When it comes to local relevance and the need to consider research performance in its specific context, we consider the liberty offered to research units of establishing their own evaluation criteria in addition to the minimum requirements demanded through legislative and normative documents, to be a 
good practice, as well as in relation to the recommendation regarding the measurement of research performance against own goals. Moreover, another good practice refers also to the fact that each research community represented through a specialized CNATDCU Commission has the possibility of establishing its own list with standards and indicators.

On the other hand, the strong focus on ISI articles, impact factor and the no. of citations in ISI articles might be detrimental to a certain degree to candidates who conducted research relevant at local level or more niched, as their research outputs might not obtain the same number of citations as those written on topics of international interest. A helpful practice in this regard can be otherwise identified in the fact that, for each such competition, a dedicated commission is set up with researchers and university professors coming from the same field, which are the ones best suited to reflect on the performance of a researcher's previous activity in light of the field in which he/ she activates.

Moreover, the way in which the competition commissions are composed - 5 members, of which 3 are part of the research unit organizing the competition, can also contribute to evaluating candidates against the research goals and mission of the research unit in case, even though this is not explicitly mentioned. In this context, we encourage including in the normative and legislative documents specifications regarding the measurement of performance against the research goals and missions of both the institutions organizing the competition, as well as of candidates. In addition, we recommend relevant authorities to explore evaluation arrangements which incorporate research activity related practices such as rigor, transparency, and reporting, alongside research outputs. The liberty offered to research units of adding new criteria in accordance with their specificities, and the fact that for each research field, the research community decide their own standards and indicators, also represent good practices which must be continued.

Further, when it comes to the next identified topic - the assessment approach, we tried to conclude what is the predominant approach of the research assessment process, and what are its potential advantages, as well as its shortcomings. 
By looking at the 2016 Order's annexes with standards and indicators, we notice a mixed approach, qualitative and quantitative. Certain thematic Commissions have included in their annex recommendations for competition commissions regarding the need of taking into account with predilection the quality of research done by candidates. However, given the fact that the respective documents do not give explicit directions regarding how this should be done, nor do they include qualitative indicators, the qualitative approach is weaker. In order to have a predominant qualitative approach, based on peer review and informed by quantitative data, clear directions regarding methodology, evaluation criteria and indicators should be included in the dedicated normative document.

In what concerns the assessment approach, we recommend that increased weight should be given to its qualitative component, by incorporating other types of indicators, more qualitative ones, which for example refer to research activity related practices as opposed to indicators solely regarding research outputs. As opposed to the approach described in the 2005 normative documents, the one from 2016 has introduced a broader range of indicators and of research outputs being taken into consideration. However, we advise for the latter to be even further diversified, by taking into consideration other types of research activities also, and by increasing the focus on the scientific content of research outputs, as well as on societal relevance and open science practices.

In the case of the topic of metrics and indicators, the focus was especially on identifying what types of such metrics and indicators are used in the assessment process, and what are their (potential) effects. Here, only the indications regarding the need to prioritize the quality of research outputs over other types of indicators illustrate a potential prioritization of scientific content over publication metrics, as the Hong Kong principles recommend. (Moher et al. 2020, 3) However, as we also argued previously, this is not enough, as they do not include specific directions or indicators for doing so. Moreover, given the focus on the number of publications, citations and funding obtained, the competition commission might get little information about the rigor and quality of research activities conducted by a researcher. In this context, Moher et al. consider that, through this kind of metrics, we also get "little information about a publication's 
(and therefore a researcher's) contribution to research and society" (Moher et al. $2020,3)$. In contrast, the authors give the example of researchers involving other relevant stakeholders in the process of developing research questions as a good practice.

To what concerns the second topic of recommendations approached here having a suite of indicators and metrics that acknowledge the variations existing across fields and types of research, we observe that the analysed evaluation arrangement in Romania provides a partially limited number of indicators, which do not cover entirely the diversity of potential research outputs and activities. As we have also stated previously, there is a strong focus on research outputs and traditional research assessment metrics, such as publication metrics. There are no references regarding the use of indicators and metrics related to research activity and process related practices, nor to indicators that reflect different types and stages of research. Indeed, the arrangement allows for additional criteria to be included by the research institutions/ units organizing the competitions, but at this stage of research it is not known exactly how the respective organizations act in this regard.

Other aspects observed in this regard refer to the use of indicators regarding membership in prestigious editorial teams, awards received, contribution brought to training of other specialist, initiation of study programs. the latter two rewarding the role of a researcher in passing on knowledge to other researchers, and all of them represent good practices in research assessment. On the other side, we took note of the lack of reference regarding Open Science practices and principles, as well as to any aspects regarding societal relevance or impact.

Here, a set of recommendations were identified based on the conducted analysis. The first one refers to exploring the possibility of using indicators which also reflect research activity related practices (such as rigor, quality etc.), and indicators that illustrate contribution brought to research and society, as opposed to a sole focus on research outputs. Secondly, we recommend developing an even broader list with indicators which reflect the variations 
existing across fields ${ }^{1}$, types, and stages of research, as well as associated research outputs, from which research organizations organizing competitions can choose the indicators that best suit their case, their missions, and goals. One example in this sense is provided through the Strategy Evaluation Protocol 20212027 from the Netherlands (35-39), which offers a list of possible indicators with guidance regarding their use. And lastly, the need to explore integrating in the research assessment arrangement indicators reflecting Open Science policies and practices, as well as societal impact/ relevance was identified.

In what concerns transparency, the following questions were used in guiding our analysis: How transparent are the evaluation approach, criteria, and specific methodology? Do researchers have access to their evaluation results? Do researchers have the right to reply?

First, the principle of transparency is not mentioned explicitly in the analysed legislative and normative documents, which also do not give any information regarding specific procedures to be followed in this regard, outside of the fact that the competition will have to be announced.

Regarding the process of data collection, candidates prepare themselves their portfolios with research outputs and other materials supporting their application. The information provided regarding what the candidates' portfolio must contain is comprehensive, and at the same time it has the potential of leaving enough room for research units to particularize the requirements depending on their needs and the specificities of the research fields in which they activate.

When it comes to candidates' access to their own detailed evaluation results and analyses, the documents do not offer specific information. In this regard, further research could be conducted on research assessment methodologies used the level of research institutions/ units to see if such practices are being implemented.

${ }^{1}$ In which case, the fact that each thematic Commission elaborates its own list with standards and indicators is already useful. 
In this case, we recommend introducing the principle of transparency in the legislative and normative documents, together with explicit information regarding how it should be translated into research assessment processes. In addition, candidates should be granted the right to view the detailed analyses used to justify the evaluation results, as well as the right to reply, candidates being able to express their potential discontent with the results obtained. Furthermore, the assessment process should be kept as open and simple as possible for transparency and efficiency reasons.

Further, we have aimed to identify whether there are any monitoring and evaluation arrangements in place regarding the results and implementation of the research assessment process, if the research assessment arrangement was revised, and what is the frequency with which revisions are being made if that is the case. These questions were used to analyse how the last approached topic is reflected in the research assessment practices from Romania - revision of assessment processes.

In this case, it is clear that there are no formal monitoring and evaluation arrangements in place for evaluating the potential effects of the research assessments conducted, nor any other studies are available on that matter. However, the Order regarding the minimum standards has suffered two major changes in time, new versions being published in 2012, respectively 2016, as a result Moreover, no special references were identified within the analysed documents regarding how potential situations of discrimination, bias or unfair treatment should be dealt with in research assessment competitions.

In this context a monitoring and evaluation mechanism should be developed at national level to keep track of results obtained, as well as of their effects. Once every couple of years, a rigorous evaluation needs to be commissioned to find out how research organizations conduct such assessments and what are the latter's effects on researchers' careers, as well as at the level of organizations and at system level. Following such evaluations, legislative and normative documents regulating the research assessment procedures at case will have to be revised and updated accordingly. 


\section{DISCUSSION}

The present analysis has focused on how senior researchers are assessed for hiring and career development in Romania, by looking at dedicated normative and legislative documents. One option for going more in-depth in this regard could consist of analysing all normative and legislative documents regulating researchers' assessment, regardless of their level of seniority. Or, even extending the research to cover how academic staff and researchers from universities are assessed also. In this way, we could also look at how the assessment practices used in the case of the two categories - researchers from research institutes versus academic faculty, compare to each other, identify potential common aspects and differences.

Moreover, the research could also be taken further by exploring in more detail the types of actions and practices that will need to be adopted for each provided recommendation, together with an analysis of their potential implications. The current research serves as a start in this regard and only touches the surface when it comes to potential effects at national level. The latter can be the subject of a separate research process.

Other topics of interest refer to the arrangements used for the assessment of research organizations such as institutes or universities, or for the assessment of applicants under specific funding lines and programs, as all such practices need periodic revision, in terms of mechanisms and effects at institutes', individual researchers' and system's levels.

\section{CONCLUSIONS}

The paper aimed at analysing and providing recommendations regarding the way in which candidates are evaluated for hiring or career advancement as senior researchers in Romania, from the perspective of recent developments regarding research assessment approaches registered at European and international levels. For this, we have first looked at existing literature on 
subjects such as what research performance and success mean, research assessment - understanding and existing approaches, evaluation criteria, indicators and metrics used - advantages, disadvantages, and potential effects.

After conducting the analysis of relevant legislative and normative documents regulating the evaluation process for candidates aspiring to become senior researchers (Scientific Researcher I and II), the following conclusions were reached.

In this specific context, research performance is understood as a of research outputs, academic impact (citations, visibility of research outputs), research management and funding obtained for research projects, as well as teaching related outputs in the case of teaching positions (courses, initiation of new study programs), from which we noticed an increased focus on the first two, especially articles published in ISI indexed journals and citations. And no special attention is given to research activity and reporting related processes, as compared to research outputs. Good practices in this regard refer to having separate Commissions regulating the standards and indicators for evaluation for each research field, the freedom offered to research units of adding their own evaluation criteria to the minimum requirements demanded through legislative and normative documents, as well as to having mixed assessment committees with members from both within the research organization, which create the premises for ensuring that the selected candidate is best suited given the goals of the organization, and from outside it, bringing new perspectives to the processes.

Recommendations regarding the way in which research performance is understood: explore evaluation arrangements that incorporate research activity related practices such as rigor, transparency, and reporting, alongside research outputs; explore introducing in the evaluation arrangement explicit specifications regarding the measurement of performance against research goals and missions of the research unit organizing the competition.

Regarding the assessment process, a mixed approach - qualitative and quantitative, was observed, as it involves both the use of peer review and of traditional metrics - publication metrics, funding obtained for research projects etc. However, there is a stronger focus on research outputs, their visibility and 
academic impact, and implicitly on the quantitative dimension. Even though certain thematic Commissions have provided together with their lists of standards and indicators the recommendation that evaluators should first consider the quality of research conducted by candidates and then to metrics, clear indications regarding how this should be done in practice, as well as dedicated indicators are missing from the normative document.

Recommendations regarding the assessment approach: Given existing recommendations at European and international levels, increased weight should be given to the qualitative component, by incorporating other types of indicators, of qualitative type, beside those that focus on research outputs. Moreover, scientific content and quality of research outputs should be prioritized, irrespective of their performance reflected through publication metrics. For this, dedicated indicators and clear indications on how they can be measured are needed.

To what concerns metrics and indicators, the following aspects were observed: a focus on research outputs and academic impact related metrics, such as publication metrics, a lack of indicators reflecting variations in types and stages of research, no reference to societal impact or contribution to society, no reference to Open Science principles and practices. In terms of good practices identified, we ought to mention that each research community decides what indicators to use; the evaluation arrangement allows for additional evaluation criteria and corresponding indicators to be used by research units organizing competitions; and indicators such as membership in prestigious editorial teams, awards received, contribution to training other specialists and the initiation of educational programs, indicate a strong focus on the international dimension of research, as well as on passing on knowledge to others.

Recommendations regarding indicators and metrics: develop an even broader list with indicators which reflect the variations existing across fields, types, and stages of research, as well as associated research outputs, from which research organizations organizing competitions can choose the indicators that best suit their case; explore introducing in this broader list, indicators reflecting Open Science practices and societal impact/ relevance. 
The principle of transparency is not mentioned explicitly anywhere in the analysed legislative and normative documents, and we consider that it should be introduced, together with explicit information regarding the way in which it should be implemented. Moreover, giving candidates access to the detailed analyses behind their evaluations and a right to reply should also be considered in this regard. The evaluation process needs to be kept as open and simple as possible for both transparency and efficiency reasons.

Other recommendations provided through the four reference documents refer to the need for periodical revisions of the assessment processes. In this context, no formal monitoring and evaluation arrangements were identified, even though the Order with minimum standards was revised twice since 2011, nor references regarding how potential situations of discrimination, bias or unfair treatment should be dealt with in research assessment competitions. Thus, we recommend for a monitoring and evaluation system to be developed in order to keep track of results obtained, and of their effects. Based on rigorous evaluations regarding how research organizations conduct such assessments and their effects at different levels - individual researchers, organizations and system, the relevant legislative and normative documents should be revised and updated accordingly.

\section{AKNOWLEDGEMENT}

This paper was financially supported by the Human Capital Operational Program 2014-2020, co-financed by the European Social Fund, under the project POCU/380/6/13/124708 no. 37141/23.05.2019, with the title "ResearcherEntrepreneur on Labour Market in the Fields of Intelligent Specialization (CERT-ANTREP)", coordinated by the National University of Political Studies and Public Administration." 


\section{REFERENCES}

- American Society for Cell Biology. 2013. San Francisco Declaration on Research Assessment. https://sfdora.org/read/.

- Aubert Bonn, Noemi and Wim Pinxten. 2021. "Rethinking success, integrity, and culture in research (part 1) - a multi-actor qualitative study on success in science". Research Integrity and Peer Review. https://doi.org/10.1186/s41073-020-00104-0.

- Aubert Bonn, Noemi and Wim Pinxten. 2021. "Rethinking success, integrity, and culture in research (part 2) - a multi-actor qualitative study on success in science". Research Integrity and Peer Review. https://doi.org/10.1186/s41073-020-00105-z.

- Bazley, Pat. 2010. "Conceptualizing research performance”. Studies in Higher Education Journal. 35, Issue 8: 889-903. https://doi.org/10.1080/03075070903348404.

- Campbell, Davicd, Michelle Picard-Aitken, Grégoire Côté, Julie Caruso, Rodolfo Valentim, Stuart Edmonds, Gregory Thomas Williams et al. 2010. "Bibliometrics as a Performance Measurement Tool for Research Evaluation: The Case of Research Funded by the National Cancer Institute of Canada" American Journal of Evaluation. 31, Issue 1: 66-83. https://journals.sagepub.com/doi/10.1177/1098214009354774.

- Elsevier. 2019. Factsheet: Salami Slicing https://www.elsevier.com/_data/assets/pdf_file/0011/653888/SalamiSlicing-factsheet-March-2019.pdf.

- European Commission. 2016. Open Innovation, Open Science, Open to the World. A Vision for Europe. doi:10.2777/061652

- European Commission. 2017. Next-generation metrics: Responsible metrics and evaluation for open science. Report of the European Commission Expert Group on Altmetrics. DOI 10.2777/337729, https://op.europa.eu/en/publication-detail/-/publication/b858d9520a19-11e7-8a35-01aa75ed71a1. 
- Foster webpage. "What is Open Science? Introduction". https://www.fosteropenscience.eu/content/what-open-scienceintroduction (last consulted on 06.09.2021).

- Hicks, Diana, Paul Wouters, Ludo Waltman, Sara de Rijcke and Ismael Rafolds. 2015. "Bibliometrics: The Leiden Manifesto for research metrics" Nature April 23, 520 (429-431). https:/ / doi.org/10.1038/520429a

- Human Genome Project Information Archive 1990-2003. Policies on Release of Human Genomic Sequence Data Bermuda-Quality Sequence. https://web.ornl.gov/sci/techresources/Human_Genome/research/berm uda.shtml (last consulted at 20.09.2021).

- Jonkers, Koen and Thomas Zacharewicz. 2016. Research Performance Based Funding Systems: a Comparative Assessment. Publications Office of the European Union: Luxembourg. doi:10.2791/70120, JRC101043.

- Law no. $319 / 2003$ from the $8^{\text {th }}$ of July, 2003, regarding the status of the research-development staff (available only in Romanian). https://www.utgjiu.ro/docs/posturi-didactice-cercetare/2020/Lege_3192003.pdf.

- Ministry of National Education and Scientific Research, Order no. 3482/ 2016 on the approval of the Regulation on the organization and functioning of the National Council for Attestation of University Degrees, Diplomas and

Certificates, https://www.edu.ro/sites/default/files/ORDIN\%203482-2016.pdf.

- Moher, David, Lex Bouter, Sabine Kleinert, Paul Glasziou, Mai Har Sham, Virginia Barbour, Anne-Marie Coriat, Nicole Foeger, Ulrich Dirnagl. 2020. "The Hong Kong Principles for assessing researchers: Fostering research integrity". Plos Biology. https://doi.org/10.1371/journal.pbio.3000737.

- OECD. 2015. "Making Open Science a Reality". OECD Science, Technology and Industry Policy Papers. No. 25. OECD Publishing: Paris. http://dx.doi.org/10.1787/5jrs2f963zs1-en.

- Order no. 5101 of October 3, 2005, for the approval of the Evaluation System regarding the conferment of the title of $2^{\text {nd }}$ degree Scientific Researcher. http://legislatie.just.ro/Public/DetaliiDocumentAfis/34952. 
- Order no. 4478 from 2011 regarding the approval of the minimum necessary and mandatory standards for conferring didactic titles in higher education, professional R\&D degrees and the habilitation certificate, for the scientific fields related to the panel P1 - Mathematics and natural sciences, P2 - Engineering, P3 - Biomedical science, CNATDCU. http://www.cnatdcu.ro/criterii/abilitare/.

- Order no. 4691 from 2011 regarding the approval of the minimum necessary and mandatory standards for conferring didactic titles in higher education, professional R\&D degrees and the habilitation certificate, for the scientific fields related to the panel P4 - Social Sciences, CNATDCU. http://www.cnatdcu.ro/wp-content/uploads/2011/04/05351-3.pdf.

- Order no. 4692 from 2011 regarding the approval of the minimum necessary and mandatory standards for conferring didactic titles in higher education, professional R\&D degrees and the habilitation certificate, for the scientific fields related to the panel P5 - Arts and Humanities, CNATDCU. http://www.cnatdcu.ro/criterii/abilitare/.

- Order no. 5100 of October 3, 2005, for the approval of the Evaluation System regarding the conferment of the title of $1^{\text {st }}$ degree Scientific Researcher, http://legislatie.just.ro/Public/DetaliiDocumentAfis/34951.

- Order no. 6560 of December 20, 2012, on the approval of the minimum necessary and obligatory standards for the conferment of didactic titles in higher education and professional degrees of research and development.

- Priem, Jason and Bradley M. Hemminger. 2010. "Scientometrics 2.0: Toward new metrics of scholarly impact on the social Web". First Monday. 15(7). http:/ / firstmonday.org/article/view/2874/2570

- Research Excellence Framework webpage. https://www.ref.ac.uk/, last consulted at 20.09.2021.

- Science Europe. 2020. Position Statement and Recommendations on Research Assessment Processes. DOI: 10.5281/zenodo.4916155

- Technopolis Group. 2019. Science Europe Study on Research Assessment Practices. Final Report. DOI:10.5281/zenodo.4915998. 
- Tripp, Simon and Martin Grueber. 2011. Economic Impact of the Human Genome Project. https://www.battelle.org/docs/defaultsource/misc/battelle-2011-misc-economic-impact-human-genomeproject.pdf.

- UKRI webpage 2021. How we're improving your funding experience. Last updated 16 April, 2021 https://www.ukri.org/apply-for-funding/howwere-improving-your-funding-experience/introducing-a-better-way-foryou-to-evidence-your-contributions/ (last consulted on 19.09.2021).

- UNESCO. 2021. Draft text of the UNESCO Recommendation on Open Science, https://unesdoc.unesco.org/ark:/48223/pf0000378381.locale=en.

- Vicente-Saez, Ruben and Clara Martinez-Fuentes. 2018. “Open Science now: A systematic literature review for an integrated definition". Journal of Business Research.

88: 428-436. https://doi.org/10.1016/j.jbusres.2017.12.043.

- VSNU, KNAW and NOW. 2020. Strategy Evaluation Protocol 2021-2027. https://www.vsnu.nl/files/documenten/Domeinen/Onderzoek/SEP_202 1-2027.pdf.

- pr, P., Rafols, I., Oancea, A., Kamerlin S.C.L., Holbrook, J.B., Jacob, M., and von Schomberg, R. (ed.). Indicator Frameworks for Fostering Open Knowledge Practices in Science and Scholarship. European Commission Independent Expert Report. July 2019. DOI: $10.2777 / 445286$. 